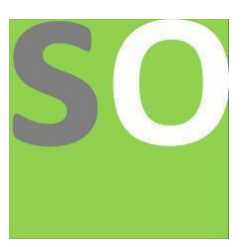

Article title: Treatment for an elderly patient with intractable postherpetic neuralgia: a case report Authors: Yan Yang[1], Junzheng Yang[2]

Affiliations: Department of Pain, Shiyan People's Hospital[1], Guangdong Nephrotic Drug Engineering Technology Research Center[2]

Orcid ids: 0000-0001-9992-3179[2]

Contact e-mail: yangjunzheng606403@163.com

License information: This work has been published open access under Creative Commons Attribution License http://creativecommons.org/licenses/by/4.0/, which permits unrestricted use, distribution, and reproduction in any medium, provided the original work is properly cited. Conditions, terms of use and publishing policy can be found at https://www.scienceopen.com/.

Preprint statement: This article is a preprint and has not been peer-reviewed, under consideration and submitted to ScienceOpen Preprints for open peer review.

DOI: 10.14293/S2199-1006.1.SOR-.PP8FFJ9.v1

Preprint first posted online: 01 February 2022

Keywords: posterior herpetic neuralgia; pain; analgesia 


\title{
Treatment for an elderly patient with intractable \\ postherpetic neuralgia: a case report
}

\author{
Abstract \\ Yan yang ${ }^{1}$, Junzheng Yang ${ }^{2 *}$ \\ ${ }^{1}$ Department of Pain, Shiyan People's Hospital (Affiliated People's Hospital of Hubei Medical \\ College), Shiyan, Hubei 442000, China \\ ${ }^{2}$ Guangdong Nephrotic Drug Engineering Technology Research Center, The R\&D Center of Drug \\ for Renal Diseases, Consun Pharmaceutic al Group, Dongpeng Avenue, No.71, Guangzhou, \\ Guangdong 510000, China. \\ Correspondence to Junzheng Yang(yangjunzheng606403@163.com), Guangdong Nephrotic Drug \\ Engineering Technology Research Center, The R\&D Center of Drug for Renal Diseases, Consun \\ Pharmaceutical Group, Dongpeng Avenue, No.71, Guangzhou, Guangdong 510000, China.
}

\begin{abstract}
Posterior herpetic neuralgia, defined as lasting pain more than one month after healing of herpes zoster, which is the most common complication of herpes zoster, and the incidence rate is the highest among the elderly population. In order to further understand the causes, pathogenesis and treatment of postherpetic neuralgia of the elderly patients, we reported a 84-year old patients with postherpetic neuralgia within three months was treated at Shiyan people's hospital by fentanyl transdermal plaster+sacral block+intradermal sensory block, and the pain symptoms were disappeared. The treatment experience may provide an evidence for clinical workers for Posterior herpetic neuralgia treatment.
\end{abstract}

Keywords: posterior herpetic neuralgia; pain; analgesia.

\section{Introduction}

Postherpetic neuralgia (PHN) is defined as the pain lasting for more than one month after the damage and healing of herpes zoster (HZ), which is one of the most common complications of herpes zoster. PHN is a kind of persistent neuralgia that is easy to reappearance, usually occurs in the back and chest, and sometimes affects the whole body ${ }^{[1]}$. The incidence rate of herpes zoster is 3-5\% per year, About $9 \%-34 \%$ of 
herpes zoster will result in PHN. The incidence and prevalence of herpes zoster and PHN increase gradually with age, about $65 \%$ of the elder patients over 60 -years old with herpes zoster will result in PHN, and the probability of occurrence of the elder patients over 60 -years old patients can reach $75 \%^{[2,3]}$.

It is necessary to apply comprehensive treatment methods such as antiviral, neurotrophic, analgesic and improving circulation in the early stage after the appearance of herpes zoster, the skin will return to normal or left pigmentation if the herpes zoster has subsided, it will enter the stage of postherpetic neuralgia if there is still severe pain including spontaneous pain and touch-induced pain. In this situation, in addition to drug treatment, nerve block, nerve root radiofrequency, epidural analgesia and spinal cord electrical stimulation were also needed to alleviate the pain. Some evidences showed that timely analgesia measures could effectively reduce the degree of pain and the course of pain within 6 months after the occurrence of postherpetic neuralgia; if the analgesic treatment was carried out after 6 months, the therapeutic effect will not be ideal.

Here we reported a 84-year old male patient with postherpetic neuralgia was admitted to Shiyan People's Hospital and the analgesic treatment was carried out within three months. Firstly the patient suffered a huge pain treated by oral administration of several drugs and sacral canal block+local injection of neurotropin, and the pain was totally relieved after treatment with fentanyl transdermal patch+sacral canal block+intradermal receptor block.

\section{Medical records}

\subsection{Medical history}

An 84-year old male patient was admitted to the department of neurology in our hospital because of "skin rash accompanied with pain on the right buttock and posterolateral thigh lasting for 2 months". He had a medical history of "spinal tuberculosis" and complained of L4-5 vertebral fusion, and denied the other history of diseases. Before admission, he took gabapentin, pregabalin, carbamazepine, ibuprofen, tramadol tablets, oxycodone, paracetamol oxycodone tablets and other analgesics through oral administration, and treated twice by sacral block+local injection of 
neurotropin had no therapeutic effect in the local hospital.

1.2 Physical examination, auxiliary examination and treatment process

After admission in our hospital, the patients were treated with circulation improvement (Xue shuan tong injection), nerve nutrition (methylprednisolone capsules, vitamin B1 tablets), pain relief (celecoxib capsules, diclofenac sodium suppositories), mood regulation (Deanxit tablets), the patient still complained of persistent discharge-like pain and paroxysmal sharp pain in the right buttock and right thigh, and the pain is unbearable.

On the sixth day of admission, the patient was consulted in our department. Physical examination found that herpes of the patient's right buttock and right thigh posterolateral skin had scab, no local ulceration and no hyperalgesia at the lesion site, with irregular paroxysmal severe pain, pain lasting for 20-30 minutes every attack, with an average of more than 10 attacks per day, and VAS score was 9 points; it is suggested that the patient should undergo lumbar CT and lumbar MRI examination to exclude the pain caused by lumbar lesions, and temporarily apply $4.125 \mathrm{mg}$ fentanyl transdermal patch to eliminate the pain caused by lumbar lesions, and considered sacral block treatment again. After 2 days of fentanyl transdermal application, the frequency of paroxysmal exacerbation of pain was significantly reduced, 2-3 times a day, but duration and degree of pain were the same as before. The results of lumbar CT showed that there were herniations of intervertebral disc at L4-5, L4-5 right facet joint and lumbar degeneration, L2-3 and L3-4 intervertebral space was narrowing; lumbar MRI examination results showed that: lumbar was degenerative, there were herniations of intervertebral disc at L4-5 and L5-S1, anterior and posterior diameter of local spinal canal was about $14 \mathrm{~mm}, \mathrm{~L} 2-4$ intervertebral space was narrowed and part of the vertebral happened fusion. Considering the degenerative change, sacral canal block+intradermal receptor block treatment was taken as follows: the patient was placed in prone position with iliac abdominal cushion, and the puncture point was in the center of sacral hiatus. the No.7 needle was used to contact the skin at a angle of $70^{\circ}-80^{\circ}$ under local anesthesia, when the sacrococcygeal ligament is broken, the needle will have a typical sense of emptiness; at this time, laid the needle body flat, 
continued to enter the needle consistent with the sacral axis for $1-2 \mathrm{~cm}$, conducted suction and resistance test to confirm that there is no blood and cerebrospinal fluid in the epidural space. Firstly injected $5 \mathrm{~mL}$ anti-inflammatory analgesic solution $(4 \mathrm{~mL}$ $2 \%$ lidocaine $+6 \mathrm{~mL} 3 \mathrm{mg}$ compound betamethasone injection solution $+10 \mathrm{~mL}$ normal saline), if there were no signs of local anesthetic poisoning or total spinal anesthesia after $5 \mathrm{~min}$ of observation, and then $15 \mathrm{~mL}$ anti-inflammatory analgesic solution was slowly injected. During the injection, the vital signs and drug reactions of the patient were closely observed, and the surrounding method was used to inject anti-inflammatory analgesic solution into the skin around the lesion to interrupt the pain nerve conduction pathway. The patient did not complain of discomfort after 30 min of observation, intradermal receptor block was taken. The treatment was taken once a week for three times.

The patient was instructed to continue to apply $4.125 \mathrm{mg}$ fentanyl transdermal patch, 3 days per patch after the first week of treatment. The patient complained of persistent acupuncture-like pain in the first 2 days after treatment with VAS score of about 4 points, and no paroxysmal aggravation of pain occurred, intermittent discharge-like severe pain occurred again on the third day, with VAS score of 6-7 points, duration of 10-20 minutes, 2-3 times per day; the patient complained of obvious pain relief, there was no severe pain but just persistent dull pain with VAS score of 3-4 after the second treatment; on the third day after the second treatment, the patient stopped fentanyl transdermal patch, there was no severe pain, the VAS score was 2-3 points; the patient complained that the pain had been relieved from the fourth week during the weekly follow-up after continuing to take gabapentin capsule $0.3 \mathrm{~g} /$ time, 3 times/day.

\section{Discussion}

The effect was not obvious when patients only took drugs for pathological neuralgia (gabapentin ${ }^{[4]}$, pregabalin, carbamazepine), non steroidal drugs (ibuprofen), opioids (tramadol, oxycodone ${ }^{[5]}$, paracetamol oxycodone tablets), or combined with sacral block and local injection of neurotropin. The treatment was also ineffective after treatment of circulation improvement (Xueshuantong injection), nerve nutrition 
(methamphetamine capsules, vitamin B1 tablets), pain relief (celecoxib capsules, diclofenac sodium suppositories), mood regulation (Deanxit tablets) in the department of neurology of our hospital. The pain was alleviated after the patient began to apply fentanyl transdermal patches. Although fentanyl transdermal patch can reduce the frequency of severe pain, it still could not reduce the intensity and duration of pain, the patient did not have paroxysmal severe pain after the combined sacral canal block+intracutaneous receptor block treatment, the patient's pain became persistent dull pain after the second treatment, the patient's pain has been relieved, the curative effect of pain should be affirmed.

Before admission, the patient had taken many kinds of analgesic drugs and mood regulating drugs, and had undergone sacral block and local injection of neurotropin twice, which could not effectively relieve the intensity, frequency and duration of pain attack; Fentanyl transdermal patch can reduce the frequency of pain attack, but it cannot reduce the frequency of pain attack and the duration of each attack. Although the intensity of fentanyl analgesia is strong, fentanyl transdermal patch alone cannot completely relieve the pain of patients. Combined with sacral canal block and intradermal receptor block, the pain of patients can be obviously alleviated, After the third sacral canalblock+intracutaneous receptor block treatment, the patient insists on taking gabapentin capsule orally, and the pain of the patient has been under effective control. The curative effect of the above method is worthy of affirmation for the treatment for elder patient with postherpetic neuralgia. Long term follow-up should be conducted in the later stage. The treatment experience of this case provides ideas and strategies for the treatment of intractable postherpetic neuralgia. In the future, similar methods can be used for treatment and clinical observation to obtain more powerful clinical evidence.

\section{Acknowledge ments}

None.

\section{Conflict of interests}

There is no conflict of interest in this article.

\section{References}


[1] Singh N. Postherpetic neuralgia. Journal of Pain and Palliative Care Pharmacotherapy[J]. 2011, 25(2): 187-189.

[2] Attal N, Lanteri-Minet M, Laurent B, et al. The specific disease burden of neuropathic pain: Results of a French nationwide survey[J]. Pain, 2011, 152: 2836-43.

[3] Doth AH, Hansson PT, Jensen MP, et al. The burden of neuropathic pain: a systematic review and meta-analysis of health utilities[J]. Pain, 2010, 149: 338-44.

[4] Ifuku M, Iseki M, Hidaka I, et al. Replacement of Gabapentin with Pregabalin in Postherpetic Neuralgia Therapy[J]. Pain Medicine, 2011, 12(7): 1112-1116.

[5] Fan BF. Postmarketing surveillance study of OxyContin ${ }^{\circledR}$ tablets for relieving moderate to severe postherpetic neuralgia pain[J]. Oncology, 2008, 74(supplement 1): 66-71. 